\title{
Development of a Stereoselective Co-mediated Dihydropyran Ring Contraction.
}

\author{
Simon J. Meek, ${ }^{\dagger}$ Fabienne Pradaux, ${ }^{\dagger}$ Emmanuel H. Demont, ${ }^{\ddagger}$ \\ and Joseph P. A. Harrity. ${ }^{* \dagger}$ \\ 'Department of Chemistry, University of Sheffield, Brook Hill, \\ Sheffield, $S 37 H F$ \\ ${ }^{*}$ GlaxoSmithKline Research and Development, New Frontiers Science Park, Third Avenue, \\ Harlow, Essex, CM19 5AW
}

\section{Supporting Information}

General. All reactions were carried out in oven $\left(130^{\circ} \mathrm{C}\right)$ or flame dried glassware under an inert atmosphere of dry $\mathrm{N}_{2}$, unless otherwise stated. Infrared (IR) spectra were recorded on a Perkin Elmer Paragon 100 FT-IR spectrometer, $\square_{\max }$ in $\mathrm{cm}^{-1}$. Bands are characterised as broad (br), strong (s), medium (m) and weak (w). ${ }^{1} \mathrm{H}-\mathrm{NMR}$ spectra were recorded on Bruker AC-250 (250 MHz), AMX-400 (400 MHz) or DRX-500 (500 MHz) spectrometers. Chemical shifts are reported in ppm from tetramethylsilane with the solvent resonance as the internal standard $\left(\mathrm{CDCl}_{3}: \square 7.26 \mathrm{ppm}\right)$. Data are reported as follows: chemical shift, multiplicity ( $\mathrm{s=singlet,} \mathrm{d}=$ doublet, $\mathrm{t}=$ triplet, $\mathrm{q}=$ quartet, $\mathrm{br}=\mathrm{broad}$, $\mathrm{m}=$ multiplet), integration, coupling constants $(\mathrm{Hz})$, and assignment. ${ }^{13} \mathrm{C}-\mathrm{NMR}$ were recorded on Bruker AC-250 (62.5 MHz), AMX-400 (100 MHz) or DRX-500 (500 MHz) spectrometers with complete proton decoupling. Chemical shifts are reported in ppm from tetramethylsilane with the solvent as the internal standard $\left(\mathrm{CDCl}_{3}: \square 77.0 \mathrm{ppm}\right)$. High-resolution mass spectrometry was obtained on a MicroMass LCT or a MicroMass Prospec. Melting points (m.p.) were recorded on a Gallenkamp melting point apparatus and are uncorrected. 
Materials. Dichloromethane $\left(\mathrm{CH}_{2} \mathrm{Cl}_{2}\right)$, acetonitrile $(\mathrm{MeCN})$ and toluene were distilled from $\mathrm{CaH}_{2}$ under an inert atmosphere of nitrogen. Tetrahydrofuran (THF) was distilled from sodium/benzophenone ketyl. Unless otherwise stated, reagents were purified prior to use following the guidelines of Perrin and Armarego. ${ }^{1}$ Analytical thin layer chromatography was performed on Merck $0.2 \mathrm{~mm}$ silica gel 60-F254 plates. Purification of reaction products was carried out by flash chromatography using $\mathrm{BDH}^{\circledR}$ silica gel (40$63 \square \mathrm{m}$ particle size). Solvents for extraction and chromatography were HPLC grade.

\section{General Procedure for the Conversion of Wittig Salts into Dihydropyran Complexes}

\section{9-14 and 21.}

Method A. $n$-BuLi (1.05 equiv) was added in a dropwise fashion to a stirring solution of the phosphonium salt (1.0 equiv) in $\operatorname{THF}(0.2 \mathrm{M})$ at $-78{ }^{\circ} \mathrm{C}$, generating a deep red colour. The red solution was allowed to stir for 5 minutes before addition of the neat aldehyde or ketone (1.1 equiv). The reaction was stirred for $2 \mathrm{~h}$ at $-78{ }^{\circ} \mathrm{C}$ unless stated otherwise, slowly warmed to room temperature and quenched by addition of water. The mixture was poured into water and extracted three times with $\mathrm{Et}_{2} \mathrm{O}$. The combined organic layers were washed with water and brine, dried over anhydrous $\mathrm{MgSO}_{4}$, filtered through a pad of celite and concentrated in vacuo.

The crude adduct was dissolved in $1 \mathrm{ml}$ of $\mathrm{CH}_{2} \mathrm{Cl}_{2}$ and treated with $20 \mathrm{ml}$ of petroleum ether before cooling to $-78{ }^{\circ} \mathrm{C}$ and decanting the organic extracts from the formed precipitates through a pad of celite and the solvent removed in vacuo. The crude enol ether in $\mathrm{CH}_{2} \mathrm{Cl}_{2}(0.2 \mathrm{M})$ was added via cannula to a $0.2 \mathrm{M}$ solution of octacarbonyldicobalt in $\mathrm{CH}_{2} \mathrm{Cl}_{2}$ at room temperature. After stirring for $1 \mathrm{~h}$ the mixture was filtered through a pad of celite, concentrated in vacuo and flash chromatographed on silica gel eluting with petroleum ether $/ \mathrm{Et}_{2} \mathrm{O} / \mathrm{Et}_{3} \mathrm{~N}(100: 10: 1)$ to afford an endo/exo mixture of pyran complexes.

To a $0.1 \mathrm{M}$ solution of the endo/exo $\mathrm{Co}_{2}(\mathrm{CO})_{6}$ complexes (1 equiv) in $\mathrm{CH}_{2} \mathrm{Cl}_{2}$ at room temperature was added pyridinium para-toluene sulphonate (0.05 equiv) portion wise.

\footnotetext{
${ }^{1}$ Perrin, D. D. and Armargeo, W. L. Purification of Laboratory Chemicals; $3^{\text {rd }}$ Ed., Pergamon Press, Oxford. 1988.
} 
After stirring for 1-3 hours 1.0 equivalent of $\mathrm{Et}_{3} \mathrm{~N}$ was added and the reaction concentrated. The resulting residue was taken up in petroleum ether (40-60) then filtered through a short pad of silica to yield the title compound.

Method B. $n$-BuLi (1.05 equiv) was added in a dropwise fashion to a stirring solution of the phosphonium salt (1.0 equiv) in THF $(0.2 \mathrm{M})$ at $-78^{\circ} \mathrm{C}$, generating a deep red colour. The red solution was allowed to stir for 5 minutes before addition of the neat aldehyde or ketone (1.1 equiv). The reaction was stirred for $2 \mathrm{~h}$ at $-78{ }^{\circ} \mathrm{C}$ unless stated otherwise, slowly warmed to room temperature and quenched by addition of water. The mixture was poured into water and extracted three times with $\mathrm{Et}_{2} \mathrm{O}$. The combined organic layers were washed with water and brine, dried over anhydrous $\mathrm{MgSO}_{4}$, filtered through a pad of celite and concentrated in vacuo.

To a $0.1 \mathrm{M}$ solution of the crude enol ether (1 equiv) in benzene at room temperature was added pyridinium para-toluene sulphonate ( 0.05 equiv) portion wise. The reaction was heated under reflux for 16-24 h then diluted with petroleum ether (40-60), filtered through a short pad of silica and the organics evaporated.

The crude residue was dissolved in $1 \mathrm{ml}$ of $\mathrm{CH}_{2} \mathrm{Cl}_{2}$ and treated with $20 \mathrm{ml}$ of petroleum ether before cooling to $-78{ }^{\circ} \mathrm{C}$ and decanting the organic extracts from the formed precipitates through a pad of celite and the solvent removed in vacuo. The crude dihydropyran in $\mathrm{CH}_{2} \mathrm{Cl}_{2}(0.2 \mathrm{M})$ was added via cannula to a $0.2 \mathrm{M}$ solution of octacarbonyldicobalt in $\mathrm{CH}_{2} \mathrm{Cl}_{2}$ at room temperature. After stirring for $1 \mathrm{~h}$ the mixture was filtered through a pad of celite, concentrated in vacuo and flash chromatographed on silica gel (eluting with a continuous gradient starting with 100:1:1 petroleum ether/Et $\mathrm{Et}_{3} \mathrm{~N} / \mathrm{Et}_{2} \mathrm{O}$ and ending with 100:10:1 petroleum ether/Et ${ }_{3} \mathrm{~N} / \mathrm{Et}_{2} \mathrm{O}$ ) to afford the title compound.

Synthesis of dicobalthexacarbonyl-6-neopentyl-2-(2-phenylethynyl)-3,4-dihydro-2Hpyran (9).

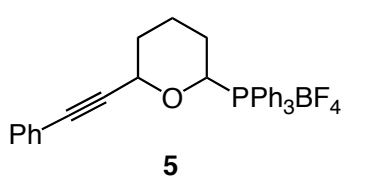

5
1) n-BuLi, THF, $-78{ }^{\circ} \mathrm{C}$;

pivalaldehyde

2) $\mathrm{Co}_{2}(\mathrm{CO})_{8}, \mathrm{CH}_{2} \mathrm{Cl}_{2}$, rt

3) $5 \mathrm{~mol} \% \mathrm{PPTS}, \mathrm{CH}_{2} \mathrm{Cl}_{2}$, rt

$33 \%$

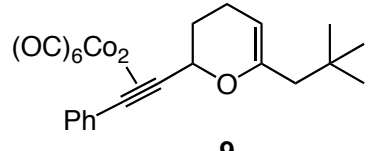

9 
Phosphonium salt $5(323 \mathrm{mg}, 0.604 \mathrm{mmol})$ was converted to the title compound according to the general procedure for dihydropyran complex formation (method A) using pivalaldehyde. The product was isolated as a dark red oil (106 mg, 33\%). ${ }^{1} \mathrm{H}$ NMR $\left(250 \mathrm{MHz}, \mathrm{CDCl}_{3}\right) \square 0.93\left(9 \mathrm{H}, \mathrm{s}, \mathrm{C}\left(\mathrm{CH}_{3}\right)_{3}\right), 1.70-1.86\left(1 \mathrm{H}, \mathrm{m}, \mathrm{CH} \mathbf{H}_{2}\right), 1.90(1 \mathrm{H}, \mathrm{d}, J=$ $\left.13.5 \mathrm{~Hz}, \mathrm{CH}_{\mathrm{a}} \mathrm{H}_{\mathrm{b}} \mathrm{C}\left(\mathrm{CH}_{3}\right)_{3}\right), 1.98\left(1 \mathrm{H}, \mathrm{d}, J=13.5 \mathrm{~Hz}, \mathrm{CH}_{\mathrm{a}} \mathbf{H}_{\mathrm{b}} \mathrm{C}\left(\mathrm{CH}_{3}\right)_{3}\right), 2.04-2.24(2 \mathrm{H}, \mathrm{m}$, $\left.\mathrm{CH}_{2}\right), 2.27-2.45\left(1 \mathrm{H}, \mathrm{m}, \mathrm{CH}_{2}\right), 4.52-4.58\left(1 \mathrm{H}, \mathrm{m}, \mathrm{CH}_{2} \mathrm{CH}=\mathrm{C}-\mathrm{O}\right), 5.08$ (1H, dd, $J=11.0$, $2.0 \mathrm{~Hz}, \operatorname{propCH}), 7.25-7.38(3 \mathrm{H}, \mathrm{m}, \mathrm{ArH}), 7.49-7.58(2 \mathrm{H}, \mathrm{m}, \operatorname{ArH}) ;{ }^{13} \mathrm{C} \mathrm{NMR}(62.5$ $\left.\mathrm{MHz}, \mathrm{CDCl}_{3}\right) \square 199.3$ (br), 153.8, 137.9, 129.7, 128.8, 127.8, 98.0, 97.3, 90.0, 75.5, 48.0, 31.0, 30.6, 29.8, 21.6; FTIR (film, $\mathrm{cm}^{-1}$ ): 3058 (w), 2956 (m), 2854 (w), 2092 (s), 2053 (s), 2022 (s), 1673 (m), 1616 (w), 1476 (w), 1443 (w), 1364 (w), 1272 (w), 1235 (m), $1046(\mathrm{~m})$; HRMS (ES) $m / z\left(\mathrm{M}^{+}+\mathrm{H}\right)$ calcd for $\mathrm{C}_{24} \mathrm{H}_{23} \mathrm{O}_{7} \mathrm{Co}_{2}$ 541.0108, found 541.0120.

\section{Synthesis of dicobalthexacarbonyl-trimethyl(2-(6-neopentyl-3,4-dihydro-2H-pyran-}

\section{2-yl)ethynyl)silane (10).}

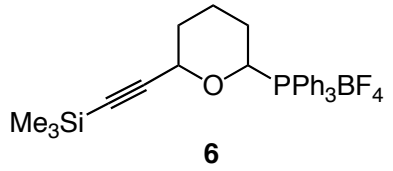

6
1) n-BuLi, THF, $-78^{\circ} \mathrm{C}$;

pivalaldehyde

2) $\mathrm{Co}_{2}(\mathrm{CO})_{8}, \mathrm{CH}_{2} \mathrm{Cl}_{2}$, rt

3) $5 \mathrm{~mol} \%$ PPTS, $\mathrm{CH}_{2} \mathrm{Cl}_{2}$, rt

$56 \%$

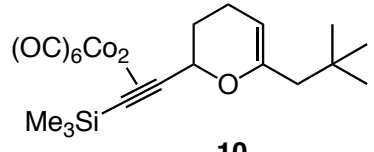

10

Phosphonium salt $6(251 \mathrm{mg}, 0.473 \mathrm{mmol})$ was converted to the title compound according to the general procedure for dihydropyran complex formation (method A) using pivalaldehdye. The product was isolated as a dark orange/red solid (142 $\mathrm{mg}, 56 \%)$. M.p. 29.0-29.5 ${ }^{\circ} \mathrm{C} ;{ }^{1} \mathrm{H}$ NMR (250 MHz, $\left.\mathrm{CDCl}_{3}\right) \square 0.30\left(9 \mathrm{H}, \mathrm{s}, \mathrm{Si}\left(\mathrm{CH}_{3}\right)_{3}\right), 0.92(9 \mathrm{H}, \mathrm{s}$, $\left.\mathrm{C}\left(\mathrm{CH}_{3}\right)_{3}\right), 1.63-2.15\left(5 \mathrm{H}, \mathrm{m}, \mathrm{CH}_{2}\right), 2.20\left(1 \mathrm{H}, \mathrm{m}, \mathrm{CH}_{2}\right), 4.46-4.54\left(1 \mathrm{H}, \mathrm{m}, \mathrm{CH}_{2}-\mathrm{CH}=\mathrm{C}-\mathrm{O}\right)$, $4.83(1 \mathrm{H}, \mathrm{dd}, J=10.5,2.0 \mathrm{~Hz}, \operatorname{propCH}) ;{ }^{13} \mathrm{C} \mathrm{NMR}\left(62.5 \mathrm{MHz}, \mathrm{CDCl}_{3}\right) \square 200.2$ (br), 153.6, 111.2, 97.8, 77.5, 75.5, 47.9, 31.6, 31.0, 29.8, 21.5, 0.8; FTIR (film, cm ${ }^{-1}$ ): 2958 (s), 2906 (m), 2866 (w), 2090 (s), 2048 (s), 2022 (s), 1673 (m), 1580 (m), 1476 (w), 1364 (w), $1302(\mathrm{w}), 1250$ (m), 1163 (w), 1048 (m); HRMS (ES) $m / z\left(\mathrm{M}^{+}+\mathrm{H}\right)$ calcd for $\mathrm{C}_{21} \mathrm{H}_{27} \mathrm{O}_{7} \mathrm{SiCo}_{2}$ 537.0190, found 537.0190.

Synthesis of dicobalthexacarbonyl-tert-butyl(3-(6-neopentyl-3,4-dihydro-2H-pyran2-yl)prop-2-ynyloxy)diphenylsilane (11). 


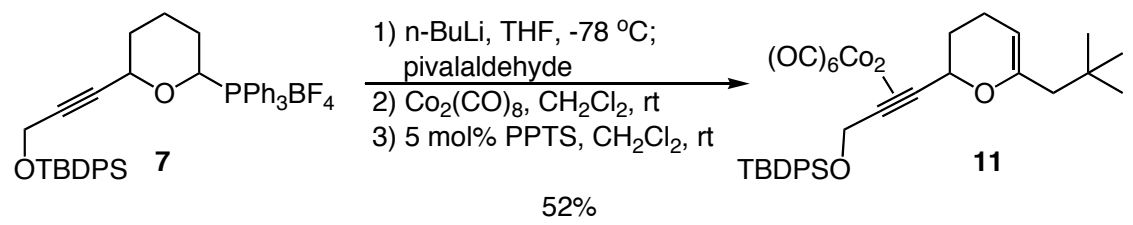

Phosphonium salt 7 (401 $\mathrm{mg}, 0.552 \mathrm{mmol})$ was converted to the title compound according to the general procedure for dihydropyran complex formation (method A) using pivalaldehdye. The product was isolated as an orange/red oil $(210 \mathrm{mg}, 52 \%) .{ }^{1} \mathrm{H}$ NMR $\left(250 \mathrm{MHz}, \mathrm{CDCl}_{3}\right) \square 0.87\left(9 \mathrm{H}, \mathrm{s}, \mathrm{Si}-\mathrm{C}\left(\mathrm{CH}_{3}\right)_{3}\right), 1.08\left(9 \mathrm{H}, \mathrm{s}, \mathrm{C}\left(\mathrm{CH}_{3}\right)_{3}\right), 1.56-1.75$ $\left(1 \mathrm{H}, \mathrm{m}, \mathrm{CH} \mathbf{H}_{2}\right), 1.81\left(1 \mathrm{H}, \mathrm{d}, J=13.5 \mathrm{~Hz}, \mathrm{CH}_{\mathrm{a}} \mathrm{H}_{\mathrm{b}} \mathrm{C}\left(\mathrm{CH}_{3}\right)_{3}\right), 1.89(1 \mathrm{H}, \mathrm{d}, J=13.5 \mathrm{~Hz}$, $\left.\mathrm{CH}_{\mathrm{a}} \mathbf{H}_{\mathrm{b}} \mathrm{C}\left(\mathrm{CH}_{3}\right)_{3}\right), 1.94-2.09\left(2 \mathrm{H}, \mathrm{m}, \mathrm{CH}_{2}\right), 2.12-2.30\left(1 \mathrm{H}, \mathrm{m}, \mathrm{CH}_{2}\right), 4.20-4.50(1 \mathrm{H}, \mathrm{m}$, $\left.\mathrm{CH}_{2}-\mathrm{CH}=\mathrm{C}-\mathrm{O}\right), 4.78(1 \mathrm{H}, \mathrm{dd}, J=10.5,2.0 \mathrm{~Hz}, \operatorname{propCH}), 4.79\left(2 \mathrm{H}, \mathrm{s}, \operatorname{propCH}_{2}\right), 7.35-$ 7.50 (6H, m, ArH), 7.67-7.76 (4H, m, ArH); ${ }^{13} \mathrm{C}$ NMR (62.5 MHz, CDCl $\left.{ }_{3}\right) \square 199.7$ (br), 153.7, 135.6, 133.0, 129.8, 127.8, 97.7, 96.2, 95.4, 75.3, 64.7, 48.0, 30.9, 30.5, 29.8, 26.6, 21.3, 19.2; FTIR (film, cm ${ }^{-1}$ ): 3073 (w), 2955 (m), 2900 (m), 2860 (m), 2094 (s), 2053 (s), 2030 (s), 1673 (m), 1612 (w), 1590 (w), 1474 (w), $1429(\mathrm{w}), 1363$ (w), 1235 (w) 1113 (m), 1054 (s); HRMS (ES) $m / z\left(\mathrm{M}^{+}+\mathrm{Na}\right.$ ) calcd for $\mathrm{C}_{35} \mathrm{H}_{38} \mathrm{O}_{8} \mathrm{NaSiCo}_{2}$ 755.0898, found 755.0889 .

\section{Synthesis of dicobalthexacarbonyl-2-(hex-1-ynyl)-6-isobutyl-3,4-dihydro-2H-pyran} (12).
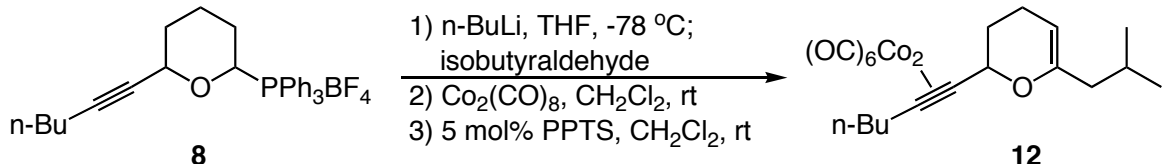

$59 \%$

Phosphonium salt $8(620 \mathrm{mg}, 1.21 \mathrm{mmol})$ was converted to the title compound according to the general procedure for dihydropyran complex formation (method A) using isobutyraldehyde. The product was isolated as a dark red oil (358 $\mathrm{mg}, 59 \%) .{ }^{1} \mathrm{H}$ NMR $\left(250 \mathrm{MHz}, \mathrm{CDCl}_{3}\right) \square 0.82-0.90\left(6 \mathrm{H}, \mathrm{m}, \mathrm{CH}\left(\mathrm{CH}_{3}\right)_{2}\right), 0.96\left(3 \mathrm{H}, \mathrm{t}, J=7.0 \mathrm{~Hz}, \mathrm{CH}_{3}\right), 1.38-$ 1.96 (8H, m, CH/CH $\mathbf{C H}_{2}, 1.98-2.14\left(2 \mathrm{H}, \mathrm{m}, \mathrm{CH}_{2}\right), 2.16-2.35\left(1 \mathrm{H}, \mathrm{m}, \mathrm{CH}_{2}\right), 2.76-2.88(2 \mathrm{H}$, $\left.\mathrm{m}, \operatorname{propCH} \mathrm{H}_{2}\right), 4.46-4.54\left(1 \mathrm{H}, \mathrm{m}, \mathrm{CH}_{2} \mathrm{CH}=\mathrm{C}-\mathrm{O}\right), 4.87(1 \mathrm{H}, \mathrm{dd}, J=10.5,2.0 \mathrm{~Hz}$, propCH); ${ }^{13} \mathrm{C}$ NMR (62.5 MHz, $\left.\mathrm{CDCl}_{3}\right) \square 200.1$ (br), 154.0, 98.5, 97.4, 95.8, 75.5, 43.6, 34.0, 33.6, 30.5, 25.9, 22.7, 22.3, 22.2, 21.3, 13.9; FTIR (film, $\mathrm{cm}^{-1}$ ): 2959 (m), 2931 (m), 2871 (w), 
2091 (s), 2049 (s), 2024 (s), 1677 (m), 1618 (w), 1466 (w), 1368 (w), 1296 (w), 1286 (w), $1233(\mathrm{w}), 1162(\mathrm{w}), 1054(\mathrm{~m})$; HRMS (ES) $\mathrm{m} / \mathrm{z}\left(\mathrm{M}^{+}+\mathrm{H}\right)$ calcd for $\mathrm{C}_{21} \mathrm{H}_{25} \mathrm{O}_{7} \mathrm{Co}_{2}$ 507.0264, found 507.0252.

Synthesis of dicobalthexacarbonyl-tert-butyl(3-(6-cyclohexyl-3,4-dihydro-2H-pyran2-yl)prop-2-ynyloxy)diphenylsilane (13).

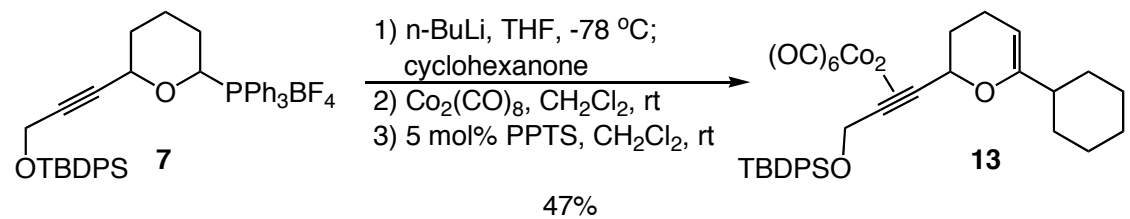

Phosphonium salt 7 (712 $\mathrm{mg}, 0.98 \mathrm{mmol})$ was converted to the title compound according to the general procedure for dihydropyran complex formation (method A) using cyclohexanone. The product was isolated as a red oil (340 mg, 47\%). ${ }^{1} \mathrm{H}$ NMR (250 $\left.\mathrm{MHz}, \mathrm{CDCl}_{3}\right) \square$ 1.00-1.32 (5H, m, $\left.\mathrm{CH}_{2}\right), 1.07$ (9H, s, C(CH $\left.)_{3}\right)$, 1.54-1.77 (6H, m, $\left.\mathrm{CH}_{2}\right)$, 1.79-2.06 (3H, m, CH/CH $\mathrm{CH}_{2}, 2.07-2.25\left(1 \mathrm{H}, \mathrm{m}, \mathrm{CH}_{2}\right), 4.40-4.48\left(1 \mathrm{H}, \mathrm{m}, \mathrm{CH}_{2}-\mathrm{CH}=\mathrm{C}-\mathrm{O}\right)$, $4.76(1 \mathrm{H}, \mathrm{dd}, J=10.5,2.0 \mathrm{~Hz}, \operatorname{propCH}), 4.79\left(2 \mathrm{H}, \mathrm{s}, \operatorname{propCH}_{2}\right), 7.33-7.49(6 \mathrm{H}, \mathrm{m}, \mathrm{ArH})$, 7.64-7.77 (4H, m, ArH); ${ }^{13} \mathrm{C}$ NMR (62.5 MHz, $\mathrm{CDCl}_{3}$ ) $\square 199.7$ (br), 159.3, 135.6, 133.0, $129.9,127.8,96.6,94.9,92.5,75.6,64.7,42.8,30.8,30.6,30.6,26.6,26.4,26.3,26.2$, 21.0, 19.2; FTIR (film, cm cm $^{-1} 3073$ (w), 2930 (s), 2856 (s), 2093 (s), 2052 (s), 2026 (s), 1673 (m), 1618 (w), 1450 (w), 1429 (w), 1362 (w), 1280 (w), 1239 (w), 1113 (m), 1058 (s); HRMS (ES) $m / z\left(\mathrm{M}^{+}+\mathrm{H}\right)$ calcd for $\mathrm{C}_{36} \mathrm{H}_{39} \mathrm{O}_{8} \mathrm{SiCo}_{2}$ 745.1078, found 745.1042.

Synthesis of dicobalthexacarbonyl-(3-(6-benzyl-3,4-dihydro-2H-pyran-2-yl)prop-2ynyloxy)(tert-butyl)diphenylsilane (14).

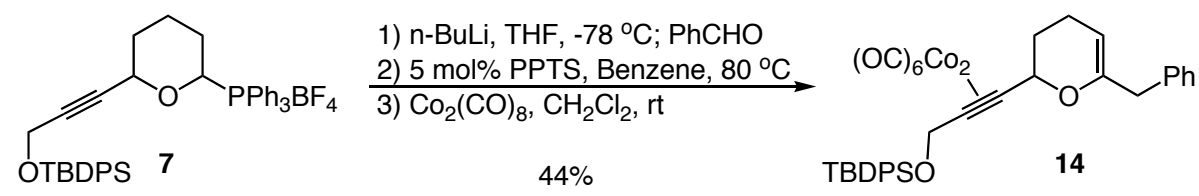

Phosphonium salt $7(342 \mathrm{mg}, 0.471 \mathrm{mmol})$ was converted to the title compound according to the general procedure for dihydropyran complex formation (method B) using benzaldehyde. The product was isolated as an orange/red oil (156 mg, 44\%). ${ }^{1} \mathrm{H}$ NMR (250 MHz, $\left.\mathrm{CDCl}_{3}\right) \square 1.07\left(9 \mathrm{H}, \mathrm{s}, \mathrm{C}\left(\mathrm{CH}_{3}\right)_{3}\right), 1.59-1.77\left(1 \mathrm{H}, \mathrm{m}, \mathrm{CH}_{2}\right), 1.92-2.26$ $\left(3 \mathrm{H}, \mathrm{m}, \mathrm{CH}_{2}\right), 3.27\left(2 \mathrm{H}, \mathrm{s}, \mathrm{CH}_{2} \mathrm{Ph}\right), 4.47-4.53\left(1 \mathrm{H}, \mathrm{m}, \mathrm{CH}_{2}-\mathrm{CH}=\mathrm{C}-\mathrm{O}\right), 4.75$ (2H, s, 
propC $\left.\mathbf{H}_{2}\right), 4.80(1 \mathrm{H}, \mathrm{dd}, J=10.5,2.0 \mathrm{~Hz}, \operatorname{propCH}), 7.06-7.19(5 \mathrm{H}, \mathrm{m}, \mathrm{ArH}), 7.35-7.50$ (6H, m, ArH), 7.68-7.76 4H, m, ArH); ${ }^{13} \mathrm{C}$ NMR (62.5 MHz, $\left.\mathrm{CDCl}_{3}\right) \square 199.7$ (br), 153.9, 138.5, 135.6, 133.0, 129.9, 128.9, 128.1, 127.8, 126.0, 96.4, 95.8, 95.2, 75.8, 64.6, 40.5, 30.2, 26.6, 21.0, 19.1; FTIR (film, $\mathrm{cm}^{-1}$ ): 3072 (w), 3030 (w), 2955 (m), 2932 (m), 2896 (m), 2858 (m), 2093 (s), 2053 (s), 2029 (s), 1677 (m), 1604 (w), 1429 (m), 1362 (m), $1284(\mathrm{w}), 1232(\mathrm{~m}), 1113(\mathrm{~s}), 1053$ (s); HRMS (ES) $\mathrm{m} / \mathrm{z}\left(\mathrm{M}^{+}+\mathrm{Na}\right)$ calcd for $\mathrm{C}_{37} \mathrm{H}_{34} \mathrm{O}_{8} \mathrm{SiCo}_{2} \mathrm{Na} 775.0585$, found 775.0598 .

\section{General Procedure for Cyclobutane Formation.}
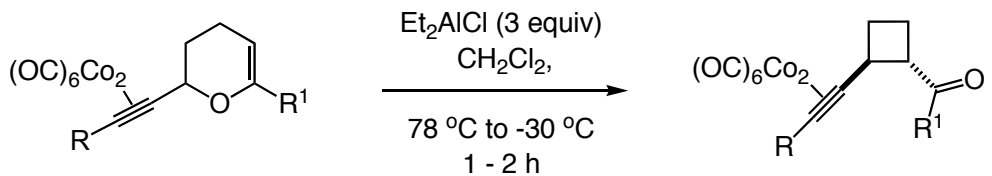

The dicobalthexacarbonyl complex was dissolved in $\mathrm{CH}_{2} \mathrm{Cl}_{2}(0.1 \mathrm{M})$ and cooled to -78 ${ }^{\circ} \mathrm{C}$. $\mathrm{Et}_{2} \mathrm{AlCl}$ (3 equiv) was then added in a dropwise fashion, after addition was complete the reaction mixture was stirred for 1 minute at $-78{ }^{\circ} \mathrm{C}$ then the reaction vessel was transferred to a $-30{ }^{\circ} \mathrm{C}$ bath and stirred for the indicated amount of time. The $-30{ }^{\circ} \mathrm{C}$ bath was removed and the reaction was warmed to $0{ }^{\circ} \mathrm{C}$ followed by addition of water. The reaction was poured onto water and extracted with $\mathrm{Et}_{2} \mathrm{O}(\mathrm{x} 3)$. The ethereal layers were combined washed with water, brine, dried over $\mathrm{MgSO}_{4}$ and concentrated. The crude product was purified by silica gel chromatography.

\section{Synthesis of dicobalthexacarbonyl-3,3-dimethyl-1-(2-(2- phenylethynyl)cyclobutyl)butan-1-one (15).}
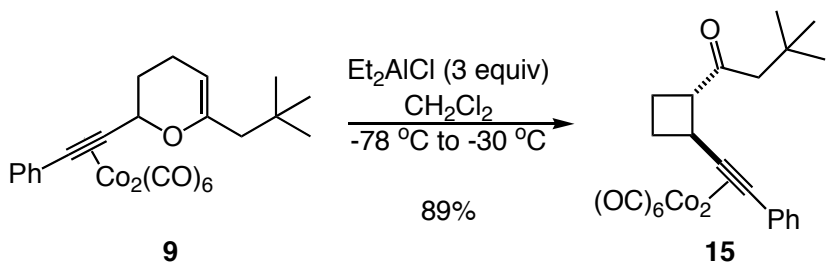

Following the general procedure, complex $9(32 \mathrm{mg}, 0.059 \mathrm{mmol})$ in $\mathrm{CH}_{2} \mathrm{Cl}_{2}$ was treated with 3 equivalents of $\mathrm{Et}_{2} \mathrm{AlCl}$ and stirred for $1 \mathrm{~h}$. Purification by column chromatography on silica gel (eluting with a continuous gradient starting with petroleum ether and ending with 10:1 petroleum ether/ $\mathrm{Et}_{2} \mathrm{O}$ ) afforded the product as a dark red solid (28 mg, 89\%). M.p. 50.1-50.4 ${ }^{\circ} \mathrm{C} ;{ }^{1} \mathrm{H}$ NMR $\left(250 \mathrm{MHz}, \mathrm{CDCl}_{3}\right) \square 0.96\left(9 \mathrm{H}, \mathrm{s}, \mathrm{C}\left(\mathrm{CH}_{3}\right)_{3}\right), 1.90-2.15(2 \mathrm{H}$, 
m, $\mathrm{CH}_{2}$ ), 2.18-2.49 (4H, m, $\mathrm{CH}_{2}$ ), 3.18 (1H, app q, $\left.J=8.5 \mathrm{~Hz}, \mathrm{R}^{1} \mathrm{R}^{2} \mathrm{CH}-\mathrm{C}(\mathrm{O})-\mathrm{CH}_{2}\right), 4.16-$ 4.30 (1H, m, propCH), 7.27-7.39 (3H, m, ArH), 7.44-7.53 (2H, m, ArH); ${ }^{13} \mathrm{C}$ NMR (100 $\left.\mathrm{MHz}, \mathrm{CDCl}_{3}\right) \square$ 209.4, 199.6 (br), 137.8, 129.5, 128.9, 127.8, 101.8, 90.9, 54.9, 53.1, 39.4, 30.7, 29.6, 26.9, 22.2; FTIR (film, $\mathrm{cm}^{-1}$ ): 3078 (w), 2955 (s), 2870 (m), 2089 (s), 2049 (s), 2016 (s), 1711 (s), 1614 (w), 1482 (m), 1443 (m), 1365 (m), 1232 (w); HRMS (ES) $m / z\left(\mathrm{M}^{+}+\mathrm{H}\right)$ calcd for $\mathrm{C}_{24} \mathrm{H}_{23} \mathrm{O}_{7} \mathrm{Co}_{2} 541.0108$, found 541.0102.

\section{Synthesis of dicobalthexacarbonyl-3,3-dimethyl-1-(2-(2-}

(trimethylsilyl)ethynyl)cyclobutyl)butan-1-one (16).

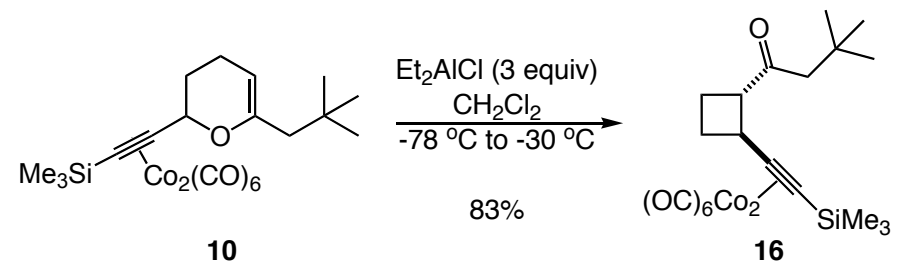

Following the general procedure, complex $10(30 \mathrm{mg}, 0.056 \mathrm{mmol})$ in $\mathrm{CH}_{2} \mathrm{Cl}_{2}$ was treated with 3 equivalents of $\mathrm{Et}_{2} \mathrm{AlCl}$ and stirred for $1 \mathrm{~h}$. Purification by column chromatography on silica gel (eluting with a continuous gradient starting with petroleum ether and ending with 10:1 petroleum ether/ $\left.\mathrm{Et}_{2} \mathrm{O}\right)$ afforded the product as a dark red oil $(25 \mathrm{mg}, 83 \%) .{ }^{1} \mathrm{H}$ NMR (250 MHz, $\left.\mathrm{CDCl}_{3}\right) \square 0.29\left(9 \mathrm{H}, \mathrm{s}, \mathrm{Si}-\mathrm{C}\left(\mathrm{CH}_{3}\right)_{3}\right), 0.99\left(9 \mathrm{H}, \mathrm{s}, \mathrm{C}\left(\mathrm{CH}_{3}\right)_{3}\right), 1.74-2.05$ $\left(2 \mathrm{H}, \mathrm{m}, \mathrm{CH}_{2}\right), 2.16-2.39$ (4H, m, $\left.\mathrm{CH}_{2}\right), 3.03\left(1 \mathrm{H}\right.$, app q, $\left.J=9.0 \mathrm{~Hz}, \mathrm{R}^{1} \mathrm{R}^{2} \mathrm{CH}-\mathrm{C}(\mathrm{O})-\mathrm{CH}_{2}\right)$, 3.95-4.09 (1H, m, propCH); ${ }^{13} \mathrm{C}$ NMR (62.5 MHz, $\mathrm{CDCl}_{3}$ ) $\square$ 208.9, 200.5 (br), 114.9, 78.4, 55.6, 53.0, 40.0, 30.7, 29.6, 27.7, 22.2 , 0.7; FTIR (film, $\mathrm{cm}^{-1}$ ): 2957 (m), 2907 (w), 2871 (w), 2087 (s), 2045 (s), 2016 (s), 1712 (m), 1584 (w), 1466 (w), 1365 (m), 1250 (m); HRMS (ES) $m / z\left(M^{+}+\mathrm{H}\right)$ calcd for $\mathrm{C}_{21} \mathrm{H}_{27} \mathrm{O}_{7} \mathrm{SiCo}_{2}$ 537.0190, found 537.0188.

\section{Synthesis of dicobalthexacarbonyl-1-(2-(3-(tert-butyldiphenylsilyloxy)prop-1-} ynyl)cyclobutyl)-3,3-dimethylbutan-1-one (17).
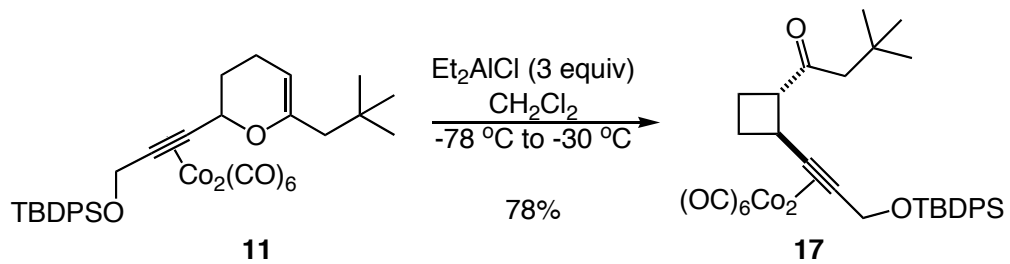
Following the general procedure, complex $11(210 \mathrm{mg}, 0.287 \mathrm{mmol})$ in $\mathrm{CH}_{2} \mathrm{Cl}_{2}$ was treated with 3 equivalents of $\mathrm{Et}_{2} \mathrm{AlCl}$ and stirred for $1 \mathrm{~h}$. Purification by column chromatography on silica gel (eluting with a continuous gradient starting with petroleum ether and ending with 10:1 petroleum ether/ $\mathrm{Et}_{2} \mathrm{O}$ ) afforded the product as an orange/red oil (163 mg, 78\%). ${ }^{1} \mathrm{H}$ NMR (250 MHz, $\left.\mathrm{CDCl}_{3}\right) \square 0.94\left(9 \mathrm{H}, \mathrm{s}, \mathrm{SiC}\left(\mathrm{CH}_{3}\right)_{3}\right), 1.08(9 \mathrm{H}, \mathrm{s}$, $\left.\mathrm{C}\left(\mathrm{CH}_{3}\right)_{3}\right), 1.75-2.07\left(2 \mathrm{H}, \mathrm{m}, \mathrm{CH}_{2}\right), 2.09-2.29\left(4 \mathrm{H}, \mathrm{m}, \mathrm{CH}_{2}\right), 3.04(1 \mathrm{H}$, app q, $J=8.5 \mathrm{~Hz}$, $\left.\mathrm{R}^{1} \mathrm{R}^{2} \mathrm{CHC}(\mathrm{O}) \mathrm{CH}_{2}\right), 3.88(1 \mathrm{H}$, app q, $J=8.5 \mathrm{~Hz}$, propCH), 4.78 (2H, s, propCH$), 7.36-$ 7.50 (6H, m, ArH), 7.69-7.78 (4H, m, ArH); ${ }^{13} \mathrm{C} \mathrm{NMR} \mathrm{(62.5} \mathrm{MHz,} \mathrm{CDCl}_{3}$ ) $\square 209.1,199.8$ (br), 135.6, 132.9, 129.9, 127.9, 100.1, 95.9, 64.6, 55.1, 52.7, 39.7, 30.7, 29.6, 27.0, 26.6, 21.9, 19.2; FTIR (film, cm cm $^{-1} 3074$ (w), 3052 (w), 2955 (s), 2896 (s), 2862 (s), 2090 (s), 2049 (s), 2027 (s), 1711 (s), 1616 (w), 1590 (w), 1474 (m), 1428 (m), 1364 (s), 1113 (s), 1066 (s); HRMS (ES) $m / z\left(\mathrm{M}^{+}+\mathrm{Na}\right)$ calcd for $\mathrm{C}_{35} \mathrm{H}_{38} \mathrm{O}_{8} \mathrm{NaSiCo}_{2} 755.0898$, found 755.0875 .

\section{Synthesis of dicobalthexacarbonyl-1-(2-(hex-1-ynyl)cyclobutyl)-3-methylbutan-1- one (18).}

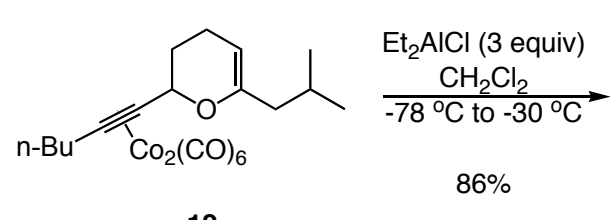

12

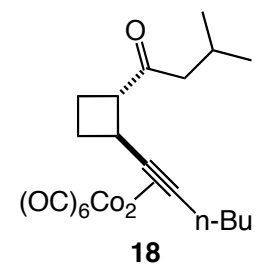

18

Following the general procedure, complex $12(355 \mathrm{mg}, 0.701 \mathrm{mmol})$ in $\mathrm{CH}_{2} \mathrm{Cl}_{2}$ was treated with 3 equivalents of $\mathrm{Et}_{2} \mathrm{AlCl}$ and stirred for $1 \mathrm{~h}$. Purification by column chromatography on silica gel (eluting with a continuous gradient starting with petroleum ether and ending with 10:1 petroleum ether/ $\mathrm{Et}_{2} \mathrm{O}$ ) afforded the product as a dark red oil (304 mg, 86\%). ${ }^{1} \mathrm{H}$ NMR (250 MHz, $\left.\mathrm{CDCl}_{3}\right) \square 0.89\left(6 \mathrm{H}, \mathrm{d}, J=6.5 \mathrm{~Hz}, \mathrm{CH}\left(\mathrm{CH}_{3}\right)_{2}\right), 0.95$ $\left(3 \mathrm{H}, \mathrm{t}, J=7.0 \mathrm{~Hz}, \mathrm{CH}_{3}\right), 1.37-1.66\left(4 \mathrm{H}, \mathrm{m}, \mathrm{CH} / \mathrm{CH}_{2}\right), 1.79-2.35\left(7 \mathrm{H}, \mathrm{m}, \mathrm{CH} / \mathrm{CH}_{2}\right), 2.73-$ $2.86\left(2 \mathrm{H}, \mathrm{m}, \operatorname{propC} \mathbf{H}_{2}\right), 3.06\left(1 \mathrm{H}\right.$, app q, $\left.J=9.0 \mathrm{~Hz}, \mathrm{R}^{1} \mathrm{R}^{2} \mathrm{HC}-\mathrm{C}(\mathrm{O}) \mathrm{CH}_{2}\right), 3.97(1 \mathrm{H}$, app q, $J=8.5 \mathrm{~Hz}$, propCH); ${ }^{13} \mathrm{C}$ NMR (100 MHz, $\left.\mathrm{CDCl}_{3}\right) \square 209.4,200.2$ (br), 101.4, 99.5, 53.9, 49.7, 39.2, 34.0, 33.8, 26.8, 24.0, 22.7, 22.6, 22.2, 13.9; FTIR (film, $\mathrm{cm}^{-1}$ ): 2961 (s), 2935 (s), 2875 (m), 2087 (s), 2044 (s), 2015 (s), 1710 (s), 1617 (w), 1467 (m), 1368 (m); HRMS (ES) $m / z\left(\mathrm{M}^{+}+\mathrm{H}\right)$ calcd for $\mathrm{C}_{21} \mathrm{H}_{25} \mathrm{O}_{7} \mathrm{Co}_{2}$ 507.0264, found 507.0263. 


\section{Synthesis of dicobalthexacarbonyl-(2-(3-(tert-butyldiphenylsilyloxy)prop-1-} ynyl)cyclobutyl)(cyclohexyl)methanone (19).

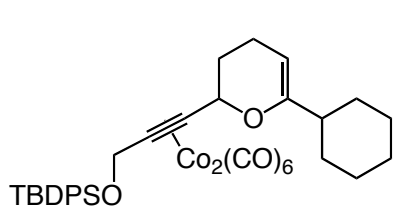

13

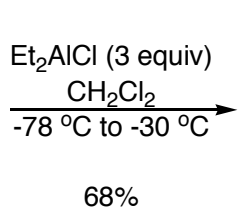

$68 \%$

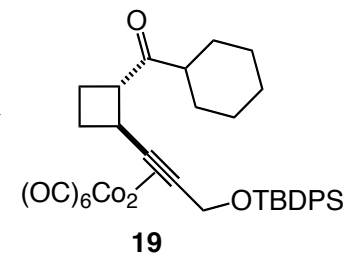

Following the general procedure, complex $13(122 \mathrm{mg}, 0.164 \mathrm{mmol})$ in $\mathrm{CH}_{2} \mathrm{Cl}_{2}$ was treated with 3 equivalents of $\mathrm{Et}_{2} \mathrm{AlCl}$ and stirred for $1 \mathrm{~h}$. Purification by column chromatography on silica gel (eluting with a continuous gradient starting with petroleum ether and ending with 10:1 petroleum ether $/ \mathrm{Et}_{2} \mathrm{O}$ ) afforded the product as an orange/red oil (83 mg, 68\%). ${ }^{1} \mathrm{H}$ NMR (250 MHz, $\left.\mathrm{CDCl}_{3}\right) \square 1.07\left(9 \mathrm{H}, \mathrm{s}, \mathrm{C}\left(\mathrm{CH}_{3}\right)_{3}\right), 1.01-1.33(4 \mathrm{H}, \mathrm{m}$, $\left.\mathrm{CH}_{2}\right), 1.55-2.04\left(8 \mathrm{H}, \mathrm{m}, \mathrm{CH} / \mathrm{CH}_{2}\right), 2.09-2.33\left(3 \mathrm{H}, \mathrm{m}, \mathrm{CH} / \mathrm{CH}_{2}\right), 3.21$ (1H, app q, $J=8.5$ $\left.\mathrm{Hz}, \mathrm{R}^{1} \mathrm{R}^{2} \mathrm{CHC}(\mathrm{O}) \mathrm{CH}_{2}\right), 3.95(1 \mathrm{H}$, app q, $J=8.5 \mathrm{~Hz}, \operatorname{propCH}), 4.76\left(2 \mathrm{H}, \mathrm{s}, \operatorname{propCH}_{2}\right)$, 7.36-7.49 (6H, m, ArH), 7.68-7.75 (4H, m, $\left.\mathrm{ArH}) ;{ }^{13} \mathrm{C} \mathrm{NMR} \mathrm{(100} \mathrm{MHz,} \mathrm{CDCl}_{3}\right) \square 212.4$, 199.8 (br), 135.5, 132.8, 129.8, 127.8, 100.0, 95.7, 64.5, 52.3, 48.9, 39.2, 28.3, 28.3, 27.3, 26.5, 25.7, 25.6, 25.6, 22.8, 19.1; FTIR (film, $\mathrm{cm}^{-1}$ ): 3073 (w), 2933 (s), 2858 (s), 2090 (s), 2048 (s), 2025 (s), 1703 (s), 1619 (w), 1590 (w), 1473 (m), 1450 (m), 1429 (m), 1363 (m), 1113 (s), 1066 (s); HRMS (ES) $\mathrm{m} / z\left(\mathrm{M}^{+}+\mathrm{Na}\right.$ ) calcd for $\mathrm{C}_{36} \mathrm{H}_{38} \mathrm{O}_{8} \mathrm{NaSiCo}_{2} 767.0898$, found 767.0905.

\section{Synthesis of dicobalthexacarbonyl-1-(2-(3-(tert-butyldiphenylsilyloxy)prop-1-} ynyl)cyclobutyl)-2-phenylethanone (20).
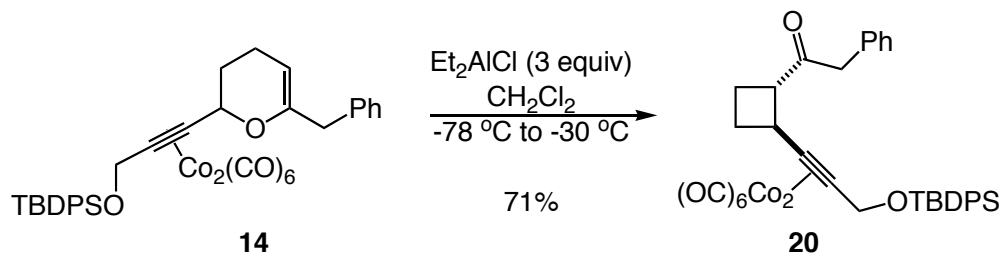

Following the general procedure, complex $14(155 \mathrm{mg}, 0.206 \mathrm{mmol})$ in $\mathrm{CH}_{2} \mathrm{Cl}_{2}$ was treated with 3 equivalents of $\mathrm{Et}_{2} \mathrm{AlCl}$ and stirred for $1 \mathrm{~h}$. Purification by column chromatography on silica gel (eluting with a continuous gradient starting with petroleum ether and ending with 10:1 petroleum ether/Et ${ }_{2} \mathrm{O}$ ) afforded the product as an orange/red oil (110 mg, 71\%). ${ }^{1} \mathrm{H}$ NMR (250 MHz, $\left.\mathrm{CDCl}_{3}\right) \square 1.00\left(9 \mathrm{H}, \mathrm{s}, \mathrm{C}\left(\mathrm{CH}_{3}\right)_{3}\right), 1.65-2.04(3 \mathrm{H}$, 
m, $\left.\mathrm{CH}_{2}\right), 2.06-2.23\left(1 \mathrm{H}, \mathrm{m}, \mathrm{CH}_{2}\right), 3.10\left(1 \mathrm{H}\right.$, app q, $\left.J=9.0 \mathrm{~Hz}, \mathrm{R}^{1} \mathrm{R}^{2} \mathrm{CH}-\mathrm{C}(\mathrm{O})-\mathrm{CH}_{2}\right), 3.53$ $\left(2 \mathrm{H}, \mathrm{s}, \mathrm{CH}_{2} \mathrm{Ph}\right), 3.91(1 \mathrm{H}$, app q, $J=8.5 \mathrm{~Hz}$, propCH), $4.68(2 \mathrm{H}, \mathrm{s}, \operatorname{propCH}), 6.98-7.06$ (2H, m, ArH), 7.12-7.23 (3H, m, ArH), 7.29-7.43 (6H, m, ArH), 7.61-7.69 (4H, m, ArH); ${ }^{13} \mathrm{C}$ NMR (62.5 MHz, $\left.\mathrm{CDCl}_{3}\right) \square$ 206.8, 199.7 (br), 135.5, 133.6, 132.9, 129.9, 129.4, 128.6, 127.8, 127.0, 99.7, 95.7, 64.5, 53.3, 48.0, 39.2, 27.3, 26.6, 23.1, 19.1; FTIR (film, $\left.\mathrm{cm}^{-1}\right): 3072$ (m), 3031 (m), 2934 (s), 2894 (s), 2860 (s), 2090 (s), 2048 (s), 2026 (s), 1706 (s), 1604 (w), 1590 (w), 1496 (w), 1473 (m), 1455 (w), 1429 (s), 1362 (m), 1113 (s), 1067 (s); HRMS (ES) $m / z\left(\mathrm{M}^{+}+\mathrm{Na}\right)$ calcd for $\mathrm{C}_{37} \mathrm{H}_{34} \mathrm{O}_{7} \mathrm{NaSiCo}_{2} 775.0585$, found 775.0564 .

\section{Synthesis of dicobalthexacarbonyl-tert-butyl(3-(6-(pent-4-enyl)-3,4-dihydro-2H-} pyran-2-yl)prop-2-ynyloxy)diphenylsilane (21).

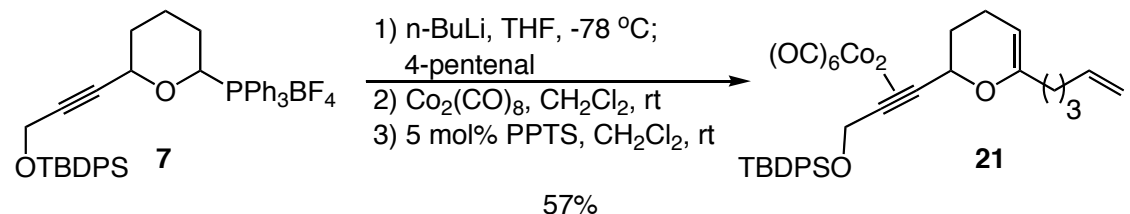

Phosphonium salt $7(601 \mathrm{mg}, 0.827 \mathrm{mmol})$ was converted to the title compound according to the general procedure for dihydropyran complex formation (method A) using 4-pentenal. The product was isolated as an orange/red oil (342 mg, 57\%). ${ }^{1} \mathrm{H}$ NMR $\left(250 \mathrm{MHz}, \mathrm{CDCl}_{3}\right) \square 1.08\left(9 \mathrm{H}, \mathrm{s}, \mathrm{C}\left(\mathrm{CH}_{3}\right)_{3}\right), 1.46-1.75\left(3 \mathrm{H}, \mathrm{m}, \mathrm{CH}_{2}\right), 1.89-2.27(7 \mathrm{H}, \mathrm{m}$, $\left.\mathrm{CH}_{2}\right), 4.46-4.53\left(1 \mathrm{H}, \mathrm{m}, \mathrm{CH}_{2}-\mathrm{CH}=\mathrm{C}-\mathrm{O}\right), 4.80\left(2 \mathrm{H}, \mathrm{s}, \operatorname{propCH}_{2}\right), 4.81(1 \mathrm{H}, \mathrm{dd}, J=10.5$, $2.0 \mathrm{~Hz}, \operatorname{propCH}), 4.87-5.01\left(2 \mathrm{H}, \mathrm{m}, \mathrm{RHC}=\mathrm{CH}_{2}\right), 5.75(1 \mathrm{H}, \mathrm{ddt}, J=17.0,10.5,6.5 \mathrm{~Hz}$, $\left.\mathrm{CH}_{2}-\mathrm{CH}=\mathrm{CH}_{2}\right), 7.36-7.51(6 \mathrm{H}, \mathrm{m}, \mathrm{ArH}), 7.68-7.79(4 \mathrm{H}, \mathrm{m}, \mathrm{ArH}) ;{ }^{13} \mathrm{C} \mathrm{NMR}(62.5 \mathrm{MHz}$, $\left.\mathrm{CDCl}_{3}\right) \square 199.7$ (br), 154.4, 138.7, 135.6, 133.0, 129.9, 127.8, 114.4, 96.4, 94.9, 94.8, 75.6, 64.6, 33.5, 33.1, 30.5, 26.6, 25.9, 21.0, 19.2; FTIR (film, $\mathrm{cm}^{-1}$ ): 3074 (w), 2932 (m), 2897 (m), 2859 (m), 2093 (s), 2052 (s), 2030 (s), 1677 (m), 1642 (w), 1616 (w), 1590 (w), 1473 (w), 1429 (m), 1362 (w), 1294 (w), 1233 (w), 1113 (s), 1056 (s); HRMS (ES) $m / z\left(\mathrm{M}^{+}+\mathrm{H}\right)$ calcd for $\mathrm{C}_{35} \mathrm{H}_{37} \mathrm{O}_{8} \mathrm{SiCo}_{2}$ 731.0922, found 731.0919 .

\section{Synthesis of dicobalthexacarbonyl-1-(2-(3-(tert-butyldiphenylsilyloxy)prop-1-} ynyl)cyclobutyl)hex-5-en-1-one (22). 


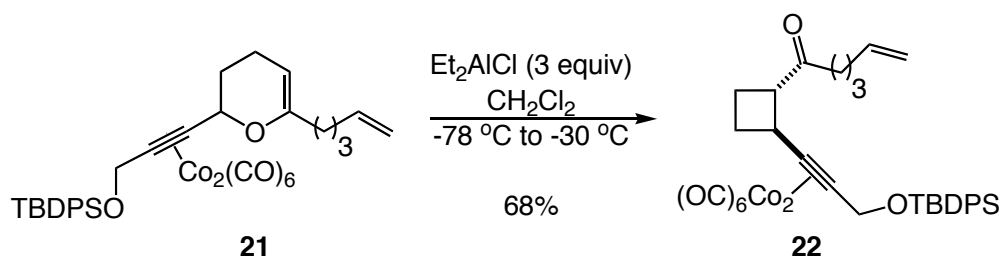

Following the general procedure, complex $21(229 \mathrm{mg}, 0.313 \mathrm{mmol})$ in $\mathrm{CH}_{2} \mathrm{Cl}_{2}$ was treated with 3 equivalents of $\mathrm{Et}_{2} \mathrm{AlCl}$ and stirred for $1 \mathrm{~h}$. Purification by column chromatography on silica gel (eluting with a continuous gradient starting with petroleum ether and ending with 10:1 petroleum ether/ $\mathrm{Et}_{2} \mathrm{O}$ ) afforded the product as a dark red oil (156 mg, 68\%). ${ }^{1} \mathrm{H}$ NMR (250 MHz, $\left.\mathrm{CDCl}_{3}\right) \square 1.07\left(9 \mathrm{H}, \mathrm{s}, \mathrm{C}\left(\mathrm{CH}_{3}\right)_{3}\right), 1.53-1.69(2 \mathrm{H}, \mathrm{m}$, $\left.\mathrm{CH}_{2}\right), 1.77-2.05\left(4 \mathrm{H}, \mathrm{m}, \mathrm{CH}_{2}\right), 2.13-2.43\left(4 \mathrm{H}, \mathrm{m}, \mathrm{CH}_{2}\right), 3.10(1 \mathrm{H}$, app q, $J=9.0 \mathrm{~Hz}$, $\left.\mathrm{R}^{1} \mathrm{R}^{2} \mathrm{CH}-\mathrm{C}(\mathrm{O})-\mathrm{CH}_{2}\right), 3.93(1 \mathrm{H}$, app q, $J=8.5 \mathrm{~Hz}$, propCH), 4.79 (2H, s, propCH$), 4.90$ $5.01\left(2 \mathrm{H}, \mathrm{m}, \mathrm{R}-\mathrm{CH}^{\mathrm{a}}=\mathrm{CH}_{2}\right), 5.71\left(1 \mathrm{H}, \mathrm{ddt}, J=17.0,10.5,6.5 \mathrm{~Hz}, \mathrm{R}-\mathrm{CH}^{\mathrm{a}}=\mathrm{CH}_{2}\right), 7.36-7.50$ (6H, m, ArH), 7.69-7.78 (4H, m, ArH); ${ }^{13} \mathrm{C} \mathrm{NMR} \mathrm{(62.5} \mathrm{MHz,} \mathrm{CDCl}_{3}$ ) $\square 209.3,199.8$ (br), $137.9,135.6$, 132.9, 129.9, 127.8, 115.2, 99.9, 95.7, 64.6, 53.7, 39.6, 39.6, 33.0, 27.2, 26.6, 22.3 22.2, 19.1; FTIR (film, cm ${ }^{-1}$ ): 3074 (m), 3052 (m), 2934 (s), 2894 (s), 2860 (s), 2090 (s), 2049 (s), 2025 (s), 1710 (s), 1642 (w), 1620 (w), 1590 (w), 1473 (m), 1429 (m), 1363 (m), 1113 (s), 1066 (s); HRMS (ES) $m / z\left(\mathrm{M}^{+}+\mathrm{Na}\right.$ ) calcd for $\mathrm{C}_{35} \mathrm{H}_{36} \mathrm{O}_{8} \mathrm{NaSiCo}_{2}$ 753.0741, found 753.0704.

\section{Synthesis of 1-(2-(3-(tert-butyldiphenylsilyloxy)prop-1-ynyl)cyclobutyl)hex-5-en-1-} one.

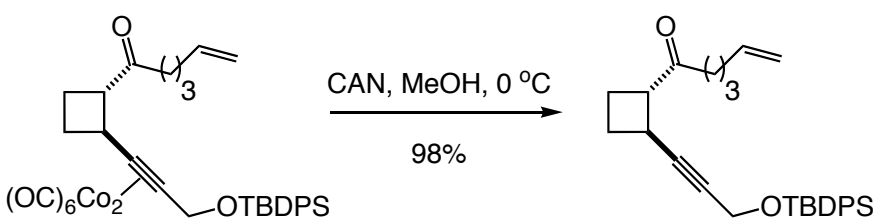

22

To a solution of cobalt complex $22(46 \mathrm{mg}, 0.063 \mathrm{mmol})$ in dry $\mathrm{MeOH}(0.63 \mathrm{~mL})$ at $0{ }^{\circ} \mathrm{C}$ was added cerium(IV) ammonium nitrate $(207 \mathrm{mg}, 0.378 \mathrm{mmol})$ in portion-wise. The reaction was stirred for $30 \mathrm{~min}$ at $0{ }^{\circ} \mathrm{C}$ then quenched by addition of water. The resulting mixture was extracted with $\mathrm{Et}_{2} \mathrm{O}(3 \times 10 \mathrm{~mL})$. The organic layers were combined, washed with brine, dried over $\mathrm{MgSO}_{4}$, filtered and evaporated in vacuo. Purification by flash column chromatography (10:1 petroleum ether: $\left.\mathrm{Et}_{2} \mathrm{O}\right)$ afforded $28 \mathrm{mg}(98 \%)$ of the enyne 
product as a clear oil. ${ }^{1} \mathrm{H}$ NMR $\left(250 \mathrm{MHz}, \mathrm{CDCl}_{3}\right) \square 1.05\left(9 \mathrm{H}, \mathrm{s}, \mathrm{C}\left(\mathrm{CH}_{3}\right)_{3}\right), 1.57-1.72$ $\left(2 \mathrm{H}, \mathrm{m}, \mathrm{CH}_{2}\right), 1.87-2.11\left(6 \mathrm{H}, \mathrm{m}, \mathrm{CH}_{2}\right), 2.39-2.30$ (2H, m, $\left.\mathrm{CH}_{2}\right), 2.97-3.17$ (2H, m, propCH, $\left.\mathrm{R}^{1} \mathrm{R}^{2} \mathrm{CH}-\mathrm{C}(\mathrm{O})-\mathrm{CH}_{2}\right), 4.35\left(2 \mathrm{H}, \mathrm{d}, J=2.0 \mathrm{~Hz}, \operatorname{propCH}_{2}\right), 4.92-5.05\left(2 \mathrm{H}, \mathrm{m}, \mathrm{CH}_{2}-\right.$ $\left.\mathrm{CH}^{\mathrm{a}}=\mathrm{CH}^{\mathrm{b}} \mathbf{H}^{\mathrm{c}}\right), 5.75\left(1 \mathrm{H}, \mathrm{ddt}, J=17.0,10.0,7.0 \mathrm{~Hz}, \mathrm{CH}_{2}-\mathrm{CH}^{\mathrm{a}}=\mathrm{CH}^{\mathrm{b}} \mathrm{H}^{\mathrm{c}}\right), 7.33-7.48(6 \mathrm{H}, \mathrm{m}$, ArH), 7.68-7.76 (4H, m, ArH); ${ }^{13} \mathrm{C}$ NMR (62.5 MHz, $\left.\mathrm{CDCl}_{3}\right) \square$ 209.5, 138.0, 135.7, 133.4, 129.7, 127.6, 115.2, 87.3, 80.4, 53.0, 51.9, 39.7, 33.1, 27.5, 26.7, 25.1, 22.5, 21.0, 19.2; FTIR (film, cm cm $\left.^{-1}\right) 3072$ (s), 3050 (s), 2932 (s), 2859 (s), 1961 (w), 1891 (w), 1827 (w), 1711 (s), 1641 (w), 1590 (w), 1473 (m), 1428 (s), 1372 (s), 1112 (s), 1376 (s); HRMS (ES) $m / z\left(\mathrm{M}^{+}+\mathrm{Na}\right.$ ) calcd for $\mathrm{C}_{29} \mathrm{H}_{36} \mathrm{O}_{2} \mathrm{NaSi}$ 467.2382, found 467.2388.

\section{Synthesis of $(E)$-7-(1-(tert-butyldiphenylsilyloxy)prop-2-en-2-yl)bicyclo[6.2.0]dec-6-} en-2-one (23) and 1-(2-(3-((tert-butyldiphenylsilyloxy)methyl)buta-1,3-dien-2-

\section{yl)cyclobutyl)hex-5-en-1-one (24).}
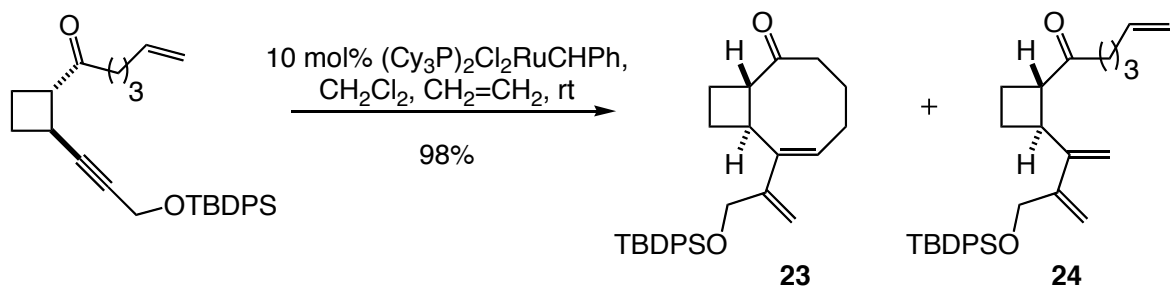

Representative procedure: To a $0.001 \mathrm{M}$ solution of the enyne $(33 \mathrm{mg}, 0.074 \mathrm{mmol}, 1.0$ equiv) in $\mathrm{CH}_{2} \mathrm{Cl}_{2}(74 \mathrm{~mL})$ was added Grubbs catalyst $25,\left(\mathrm{Cy}_{3} \mathrm{P}\right)_{2} \mathrm{Cl}_{2} \mathrm{RuCHPh},(6 \mathrm{mg}$, $0.0074 \mathrm{mmol}, 0.1$ equiv). The golden brown solution was purged with ethylene, then stirred at room temperature under a balloon of ethylene for 24 hours. The reaction was then concentrated in vacuo to give a crude brown oil. Purification by flash chromatography on silica gel (eluting with a continuous gradient starting with petroleum ether and ending with 10:1 petroleum ether/ $\mathrm{Et}_{2} \mathrm{O}$ ) afforded bicylo[6.2.0]decane 23 (22 $\mathrm{mg}, 67 \%)$ and triene $24(11 \mathrm{mg}, 31 \%)$ as clear oils. Bicyclo[6.2.0]decane 23: ${ }^{1} \mathrm{H}$ NMR $\left(500 \mathrm{MHz}, \mathrm{CDCl}_{3}\right) \square 1.07\left(9 \mathrm{H}, \mathrm{s}, \mathrm{C}\left(\mathrm{CH}_{3}\right)_{3}\right), 1.46-1.79\left(5 \mathrm{H}, \mathrm{m}, \mathrm{CH}_{2}\right), 2.02-2.20(3 \mathrm{H}, \mathrm{m}$, $\left.\mathrm{CH}_{2}\right), 2.33\left(1 \mathrm{H}, \mathrm{ddd}, J=12.5,5.5,5.5 \mathrm{~Hz}, \mathrm{CH}^{\mathrm{a}} \mathrm{H}^{\mathrm{b}}-\mathrm{C}=\mathrm{O}\right), 2.42(1 \mathrm{H}, \mathrm{ddd}, J=12.5,10.5$, $\left.5.5 \mathrm{~Hz}, \mathrm{CH}^{\mathrm{a}} \mathbf{H}^{\mathrm{b}}-\mathrm{C}=\mathrm{O}\right), 3.04-3.13(2 \mathrm{H}, \mathrm{m}, \mathrm{CH}=\mathrm{CR}-\mathrm{CH}-\mathrm{CH}-\mathrm{C}=\mathrm{O}), 4.08-4.17\left(2 \mathrm{H}, \mathrm{m}, \mathrm{CH}_{2}-\right.$ OTBDPS), 4.93-4.95 (1H, m, $\left.\mathbf{H}^{\mathrm{a}} \mathrm{H}^{\mathrm{b}} \mathrm{C}=\mathrm{CR}-\mathrm{CH}_{2}-\mathrm{OTBDPS}\right), 5.38(1 \mathrm{H}, \mathrm{dd}, J=4.0,2.0 \mathrm{~Hz}$, $\left.\mathrm{H}^{\mathrm{a}} \mathbf{H}^{\mathrm{b}} \mathrm{C}=\mathrm{CR}-\mathrm{CH}_{2}-\mathrm{OTBDPS}\right), 5.46\left(1 \mathrm{H}, \mathrm{ddd}, J=10.0,7.0,1.0 \mathrm{~Hz}, \mathrm{CH}-\mathrm{CR}=\mathrm{CH}-\mathrm{CH}_{2}\right)$, 7.35-7.44 (6H, m, ArH), 7.66-7.70 (4H, m, ArH); ${ }^{13} \mathrm{C}$ NMR (125 MHz, $\left.\mathrm{CDCl}_{3}\right) \square 211.6$, 
148.0, 143.2, 135.6, 133.5, 129.7, 127.7, 125.7, 111.2, 65.8, 55.4, 42.6, 40.0, 26.9, 25.5, 23.0, 21.5, 19.3, 18.4; FTIR (film, $\mathrm{cm}^{-1}$ ): 3072 (m), 3049 (m), 2931 (s), 2857(s), 1961 (w), 1894 (w), 1823 (w), 1706 (s), 1628 (w), 1590 (w), 1472 (m), 1462 (m), 1446 (m), $1428(\mathrm{~m}), 1362$ (m), 1112 (s); HRMS (ES) $m / z\left(\mathrm{M}^{+}+\mathrm{H}\right)$ calcd for $\mathrm{C}_{29} \mathrm{H}_{37} \mathrm{O}_{2} \mathrm{Si} 445.2563$, found 445.2547 .

Triene 24: ${ }^{1} \mathrm{H}$ NMR (250 MHz, $\left.\mathrm{CDCl}_{3}\right) \square 1.05\left(9 \mathrm{H}, \mathrm{s}, \mathrm{C}\left(\mathrm{CH}_{3}\right)_{3}\right), 1.56-1.83\left(2 \mathrm{H}, \mathrm{m}, \mathrm{CH}_{2}\right)$, 1.90-2.17 (5H, m, CH $)$, 2.21-2.49 (3H, m, CH $), 3.11-3.26\left(1 \mathrm{H}, \mathrm{m}, \mathrm{H}_{2} \mathrm{C}=\mathrm{CR}-\mathrm{CH}-\mathrm{CH}-\right.$ $\mathrm{C}=\mathrm{O}), 3.36-3.51\left(1 \mathrm{H}, \mathrm{m}, \mathrm{H}_{2} \mathrm{C}=\mathrm{CR}-\mathrm{CH}-\mathrm{CH}-\mathrm{C}=\mathrm{O}\right), 4.28-4.34$ (2H, m, $\left.\mathrm{CH}_{2}-\mathrm{OTBDPS}\right)$, 4.85-4.90 (1H, m, $\left.\mathrm{R}^{1} \mathrm{R}^{2} \mathrm{C}=\mathrm{CH}_{2}\right)$, 4.91-5.06 (3H, m, $\left.\mathrm{CH}_{2}-\mathrm{CH}=\mathrm{CH}_{2}, \mathrm{R}^{1} \mathrm{R}^{2} \mathrm{C}=\mathrm{CH}_{2}\right), 5.10-$ $5.15\left(1 \mathrm{H}, \mathrm{m}, \mathrm{R}^{1} \mathrm{R}^{2} \mathrm{C}=\mathrm{CH}_{2}\right), 5.42-5.47\left(1 \mathrm{H}, \mathrm{m}, \mathrm{R}^{1} \mathrm{R}^{2} \mathrm{C}=\mathrm{CH}_{2}\right), 5.74(1 \mathrm{H}, \mathrm{ddt}, J=17.0,10.0$, $\left.6.5 \mathrm{~Hz}, \mathrm{CH}_{2}-\mathrm{CH}=\mathrm{CH}_{2}\right), 7.33-7.46(6 \mathrm{H}, \mathrm{m}, \mathrm{ArH}), 7.63-7.71(4 \mathrm{H}, \mathrm{m}, \mathrm{ArH}) ;{ }^{13} \mathrm{C}$ NMR $(62.5$ $\left.\mathrm{MHz}, \mathrm{CDCl}_{3}\right) \square 211.1,147.5,144.4,138.0,135.5,133.5,129.6,127.7,115.1,111.8$, 109.4, 64.5, 49.5, 40.4, 40.4, 33.1, 26.8, 25.1, 22.5, 21.1, 19.3; FTIR (film, cm ${ }^{-1}$ ): 3072 (m), 3050 (m), 2932 (s), 2893 (s), 2858 (s), 1960 (w), 1901 (w), 1825 (w), 1708 (s), 1641 (w), 1599 (m), 1472 (m), 1428 (s), 1391 (m), 1362 (m), 1112 (s); HRMS (ES) m/z (M+ $\mathrm{Na}$ ) calcd for $\mathrm{C}_{31} \mathrm{H}_{40} \mathrm{O}_{2} \mathrm{NaSi} 495.2695$, found 495.2679. 